\title{
Frog skin, time series, and other kinds of data: Nonisomorphic representation and analysis
}

\author{
DARRELL L. BUTLER \\ Ball State University, Muncie, Indiana
}

\begin{abstract}
Representational art is spatially congruent, or isomorphic, with objects in the world. In other words, they are the same in some important way. But not all representation is isomorphic. Data graphs are a type of nonisomorphic representation. A major goal of data graphs is to facilitate the rapid comprehension of differences or relations. This is done by providing a kind of analogy between something that can be "seen" (the relative heights of points) and some relevant information. This article discusses some rules about data graphing, then some new visualization tools are briefly critiqued.
\end{abstract}

Representational art, sketches, and mechanical drawings are examples of depictions that represent spatial aspects of the world using spatial variables on a surface. The drawing may differ from the real-world objects in scale, detail, or color, but the object in the world and the representation are meant to be spatially congruent, or isomorphic. In other words, the depiction is spatially similar to the real object in ways that lead to similarity in the perceptions of the real object and the depicted one.

Sometimes the methods used for creating a representation do not result in an isomorphic depiction. Figure 1 shows two depictions of a frog's skin. The top one shows the skin peeled from the animal and laid flat. The perception of the bottom one is unquestionably more congruent to the real object than is the perception of the top one.

When a data graph is created from scientific data, the result is a kind of nonisomorphic representation. One represents nonspatial variables about the world (e.g., reaction time, percent correct, and frequency) as spatial variables (e.g., horizontal position and vertical position). The purpose is often to facilitate the rapid comprehension of differences, relations, and other characteristics of data.

While I will not attempt to describe a complete theory of data graphing here, I would like to comment briefly on one rule of nonisomorphic representation of data: When an attribute to be perceived is quantitative and continuous (e.g., linearity of a relationship or amount of difference between groups), then quantitative and continuous perceptual variables (also called prothetic by Stevens \& Galanter, 1957) should be used in the graph. Although I do not have any examples of this problem in the psychology literature, many newscasters who show weather maps appear to be totally ignorant of this rule. They regularly use hue to indicate temperature. Hue is not perceived as a unidimensional, quantitative, continuous variable. If brightness differences were used instead of hue, comprehension would be better facilitated.

Correspondence should be addressed to D. L. Butler, Department of Psychological Sciences, Ball State University, Muncie, IN 47306.

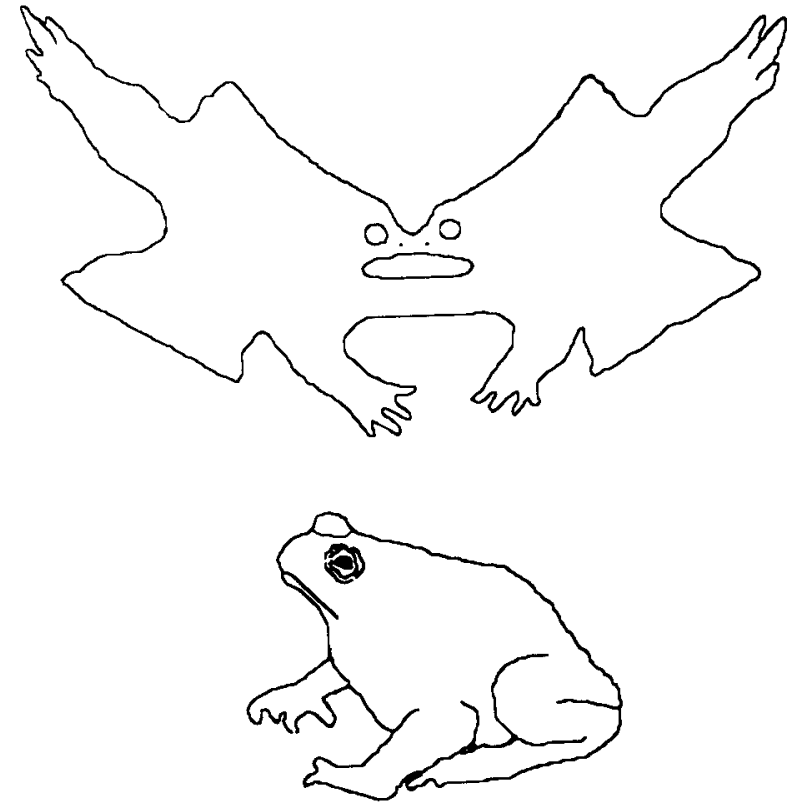

Figure 1. Two drawings of a frog's skin. The top drawing ithustrates the stin peeled from a frog and bid out fint. The bottom drawing is a more conventional representation. (These drawings were adapted from ones that were reprinted in Tufte, 1990.)

Historians have some difficulty establishing when the nonisomorphic depiction of variables and data was first done (see Beniger \& Robyn, 1978). One of the first uses was the depiction of the inclinations of the planetary orbits as a function of time. This time series, shown here in Figure 2, is from the 10th or 11th Century. Although this general approach is old, it is not irrelevant to psychology. In an analysis of animal behavior, Washburn (1992) plots a distance (i.e., the difference between response path and theoretical path) at various times, quite analogously to the time series shown here.

Those who have written about the history of data graphics (Brinton, 1939; Fienberg, 1979; Funkhouser, 1937; 


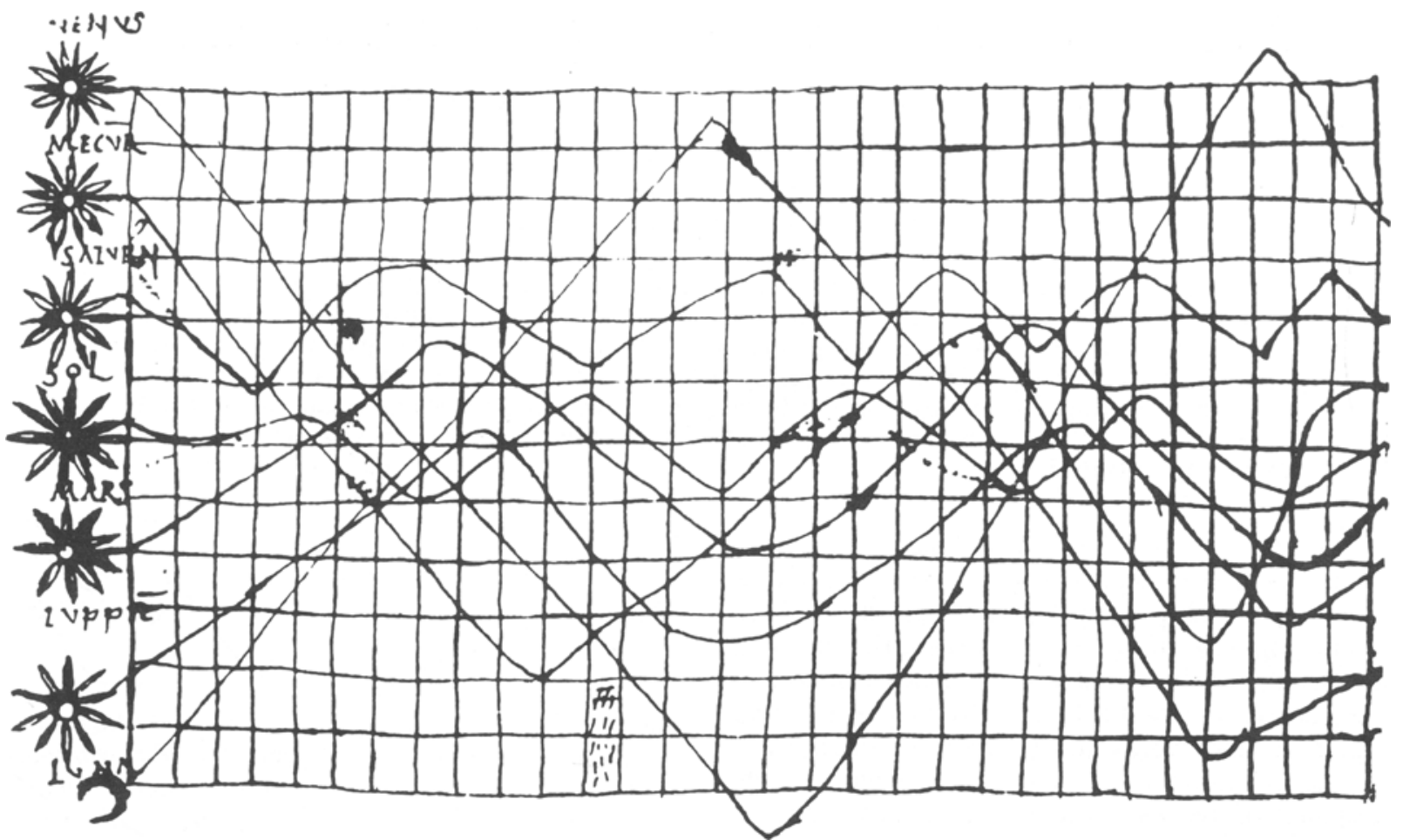

Figure 2. Planetary orbits as a function of time. This graph was created during the 10th or 11th Century. It has been reprinted in a number of modern sources (e.g., Beniger \& Robyn, 1978; Tufte, 1983).

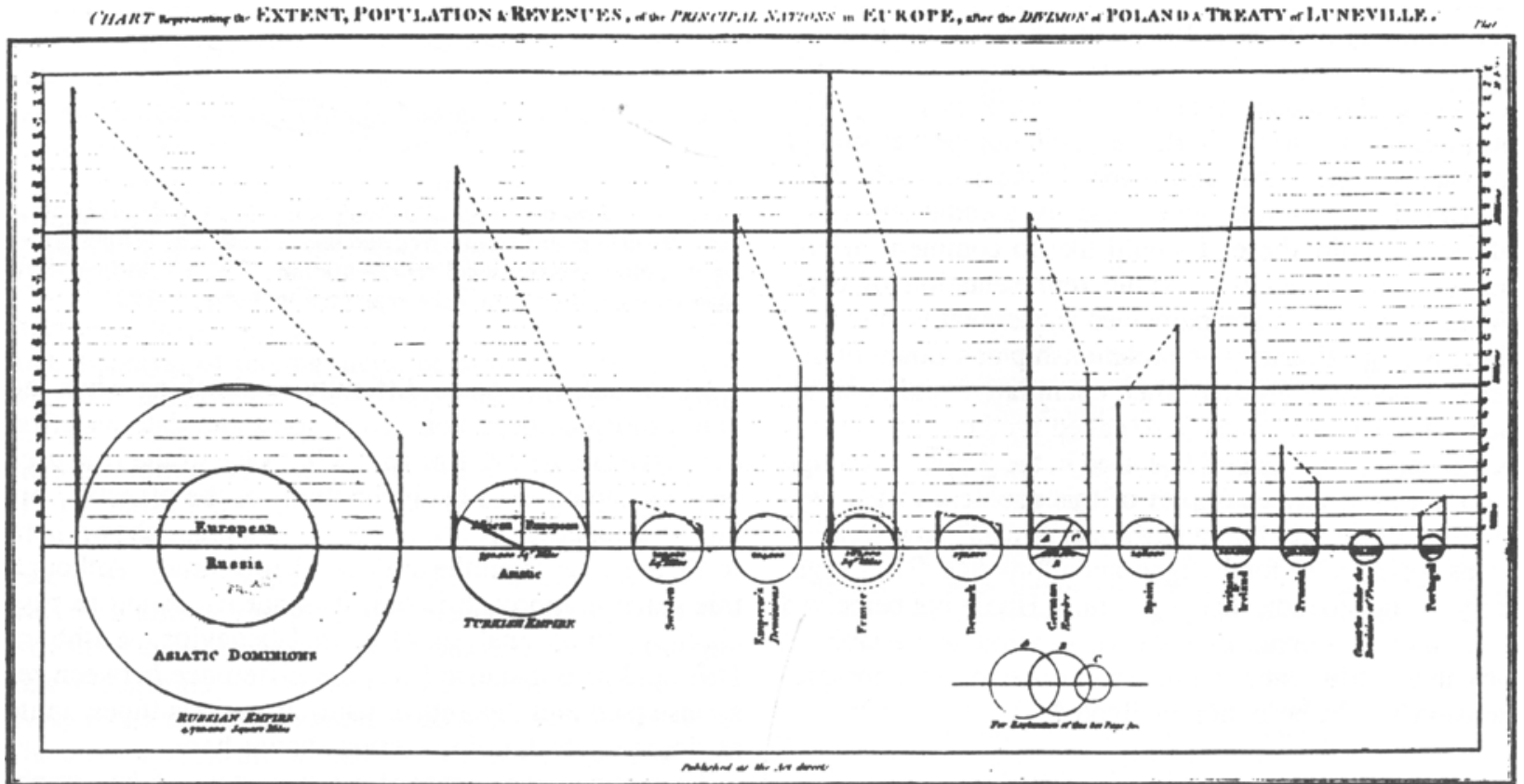

Figure 3. A multivariate data graph created by Playfair (1801) and represented in modern sources such as Tufte (1983). 


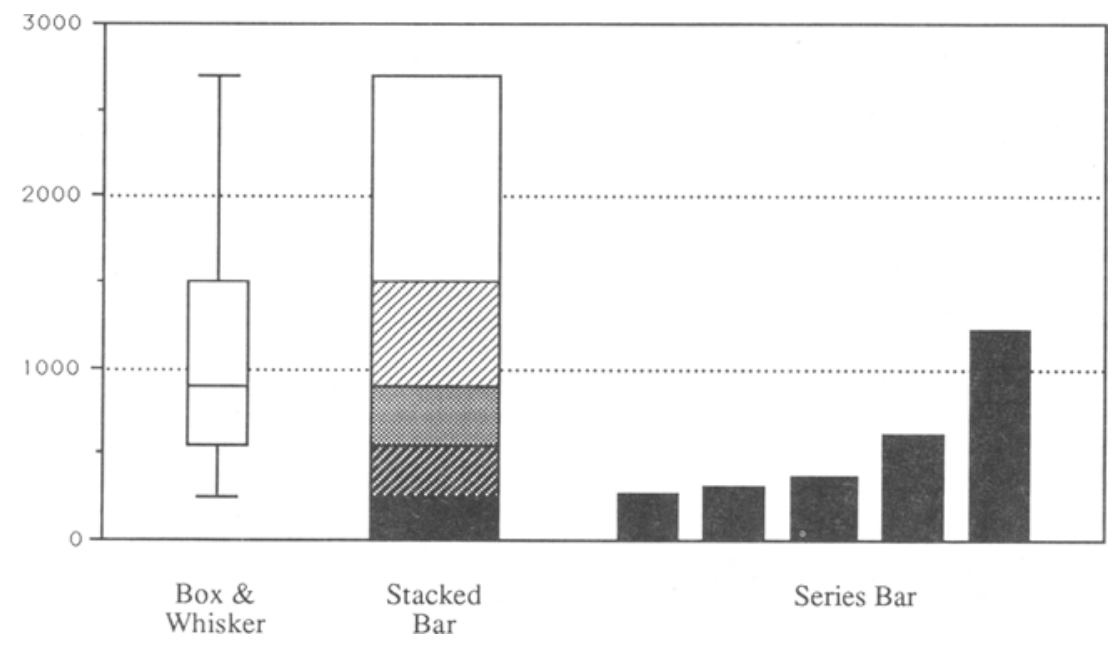

Figure 4. Several varieties of box plots of the same data.

Tufte, 1983; Wainer \& Thissen, 1981) agree that the evolution of graphs and charts of data was dramatically accelerated by Playfair. He was the first to publish a variety of time series involving economic and political variables (Playfair, 1786), the first to publish a bar chart (Playfair, 1786), the first to publish a pie chart (Playfair, 1801), and one of the first to publish depictions of multivariate data (Playfair, 1801). One of his attempts to show multivariate data is shown in Figure 3. In this figure, the area of each circle represents the size of a European country in square miles, the vertical line on the left of each circle represents the population size, and the vertical line on the right of each circle represents the revenue of the country.

In a recent review, Wainer and Thissen (1981) argue that the quality of data graphs has not increased since the time of Playfair's inventions. That makes a certain amount of sense, especially if one were to define Playfair's invention as the depiction of nonspatial variables using spatial dimensions. However, I would like to argue that the visual presentation of data and the related analytic tools have evolved in important ways.

One of the most obvious changes is that graphs can be created much more quickly today. At the time of Playfair

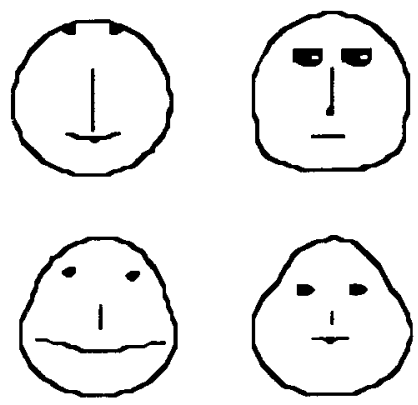

Figure 5. Examples of Chernoff faces. (the late 18th Century), all graphs were produced by hand. In this context, it is worth noting that the technologies needed to make inexpensive paper did not evolve until the 1840s. Thus, during Playfair's time, paper was expensive and people had to be especially careful during preparation of a graph. In contrast, computers today enable scientists to rapidly produce a wide variety of graphs for data exploration, analysis, or presentation of findings.

Another characteristic of the evolution of data graphs is that there are greater variations of data graphs today than at the time Playfair made his contributions. One variation of bar chart is the 5-point plot, or box-and-whiskers plot (Tukey, 1977), an example of which is shown in Figure 4 . In a box-and-whiskers plot, the middle $50 \%$ of the data is indicated with a box, the median is indicated with a line across the box, and the minimum and maximum are shown as whiskers extending from the box. There are, of course, other ways these data can be presented, as shown in Figure 4. The point is not that Tukey's approach is better, but that it is a variation that may be more desirable under some circumstances. Another variation is Chernoff's faces (Chernoff, 1973), some examples of which are shown in Figure 5. The magnitude of features in schematic faces represents the measure of variables in multivariate data. For example, one data variable may be indicated by the size of the eyes and another variable may be indicated by the length of the mouth. These multivariate plots are, in principle, the same as Playfair's, but they are a variation that may be particularly useful in some cases. Perhaps the most modern variation is the addition of motion to displays. For example, MacSpin permits rotation of three-dimensional scatterplots (see Reidbord \& Redington, 1992) and PHASER (Townsend, 1992) allows one to move toward and through a representation. Recent research is directed at determining whether there may be some kinds of information that can be extracted better from such dynamic representations (Marchak \& Zulager, 1992). 
Not only has the speed of preparing graphs decreased and the variety of graphic forms increased, but the conceptual tools for analytically processing data graphs has evolved dramatically. Consider time series. For many years, scientists relied on simple linear regression, then later, multiple regression, to extract polynomial relations from time series. Other trend-analysis systems have been used; Fourier analysis is probably the most common. But new techniques are emerging, giving scientists new tools for series analysis. Broadbent and Maksik (1992) have explored Walsh-function analysis, which decomposes trends into rectangular functions rather than sinusoids or polynomials. I am particularly intrigued by this approach and suspect it will be useful in a variety of situations. Pevey, McDowell, and Kessel (1992) discuss an informationtheory approach to quantify interevent dependencies. Information theory is not as popular as it once was, and some researchers may have difficulty ignoring the lack of popularity when they evaluate this approach. Townsend (1992) describes some software that aids scientific exploration of nonlinear dynamic systems. I believe such tools will be necessary in the evolution of psychological theory, but I am not sure many psychologists currently have the knowledge to use these packages as effectively as they use linear approaches. It is to be hoped that the kinds of software that Townsend has described will encourage and facilitate the learning of nonlinear concepts and tools.

This paper has emphasized visual processing of information, but the principle of nonisomorphic representation can be applied to the auditory system as well. The work presented by Flowers and Hauer (1992) on representing data as tones may be the seeds of a data-representation system as powerful and useful as that explored by Playfair. The potential here is still unknown but fascinating. At some future time, we may have a theory of nonisomorphic representation that can guide the development of tools not only for vision and audition but for the minor senses as well.

\section{REFERENCES}

BENIGER, J. R., ROBYN, D. L. (1978). Quantitative graphics in statistics: A brief history. American Statistician, 32, 1-11.

Brinton, W. W. (1939). Graphic presentation. New York: Brinton.

Brondbent, H. A., MAKsik, Y. A. (1992). Analysis of periodic data using Walsh functions. Behavior Research Methods, Instruments, \& Computers, 24, 238-247.

Chernoff, H. (1973). The use of faces to represent points in k-dimensional space graphically. Journal of the American Statistical Association, 68, 361-368.

FIENBERG, S. T. (1979), Graphical methods in statistics. American Statistician, 33, 165-178.

Flowers, J. H., HAUER, T. A. (1992). The ear's versus the eye's potential to assess characteristics of numeric data: Are we too visuocentric? Behavior Research Methods, Instruments, \& Computers, 24, 258-264.

FUNKHOUSER, H. G. (1937). Historical development of the graphical representation of statistical data. Osiris, 3, 269-404.

MarchaK, F. M., \& Zulager, D. D. (1992). The effectiveness of dynamic graphics in revealing structure in multivariate data. Behavior Research Methods, Instruments, \& Computers, 24, 253-257.

Pevey, M. E., McDowell, J. J., Kessel, R. (1992). Shaw's stored information as a quantitative measure of sequential structure. Behavior Research Methods, Instruments, \& Computers, 24, 228-237.

PlayfaIR, W. (1786). The commercial and political atlas. London: Corry. Playfair, W. (1801). The statistical breviary. London: Corry.

Reidbord, S. P., \& Redington, D. J. (1992). Analysis and visualization of complex psychophysiological data: Coordinated use of multiple applications on a microcomputer. Behavior Research Methods, Instruments, \& Computers, 24, 213-218.

SteVENs, S. S., \& GAlANTER, E. (1957). Ratio scales and category scales for a dozen perceptual continua. Journal of Experimental Psychology, 54, 377-411.

TOWNSEND, J. T. (1992). Don't be fazed by PHASER! Beginning exploration of a cyclical motivational system. Behavior Research Methods, Instruments, \& Computers, 24, 219-227.

TUFTE, E. R. (1983). The visual display of quantitative information. Cheshire, CT: Graphics Press.

TUFTE, E. R. (1990). Envisioning information. Cheshire, CT: Graphics Press.

TUKEY, J. W. (1977). Exploratory data analysis. Reading, MA: Addison-Wesley.

Wainer, H., Thissen, D. (1981). Graphical data analysis. Annual Review of Psychology, 32, 191-241.

WASHBURN, D. A. (1992). Analyzing the path of responding in mazesolving and other tasks. Behavior Research Methods, Instruments, \& Computers, 24, 248-252. 